Journal of Organometallic Chemistry, 301 (1986) 79-90

Elsevier Sequoia S.A., Lausanne - Printed in The Netherlands

\title{
CARBONYLCOBALT(I) COMPLEXES. THE CRYSTAL AND MOLECULAR STRUCTURE OF $\left[\mathrm{Co}(\mathrm{CO})(\mathrm{dppm})_{2}\right] \mathrm{ClO}_{4}$
}

C. CARRIEDO, P. GOMEZ-SAL, P. ROYO ${ }^{\star}$,

Departamento de Quimica Inorgánica, Universidad de Alcalá de Henares (Spain)

S. MARTINEZ-CARRERA and S. GARCIA-BLANCO

Departamento de Rayos $X$, Instituto Rocasolano CSIC, Madrid (Spain)

(Received July 29th, 1985)

\section{Summary}

New anionic carbonylcobalt(I) complexes $\left[\mathrm{X}_{2} \mathrm{Co}(\mathrm{CO})_{2}\left(\mathrm{PPh}_{3}\right)\right]\left(\mathrm{PR}_{4}\right)(\mathrm{X}=\mathrm{Cl}$, $\left.\mathrm{PR}_{4}=\mathrm{PBzPh}_{3}(\mathrm{I}) ; \mathrm{X}=\mathrm{Br}, \mathrm{PR}_{4}=\mathrm{PEtPh}_{3}(\mathrm{II})\right)$ have been prepared by reduction of the cobalt(II) halides with $\mathrm{NaBH}_{4}$ in the presence of $\mathrm{PPh}_{3}$ and the phosphonium salt $\mathrm{PR}_{4} \mathrm{X}$. Cleavage of halide bridges in dimeric or polymeric $\left[\mathrm{XCo}\left(\mathrm{PPh}_{3}\right)_{2}\right]_{n}$ and $\left[\mathrm{XCo}\left(\mathrm{PPh}_{3}\right)\right]_{n}$ gives the neutral dicarbonyl derivatives $\mathrm{XCO}(\mathrm{CO})_{2}\left(\mathrm{PPh}_{3}\right)_{2}$. Treatment of $\mathrm{ClCo}(\mathrm{CO})_{2}\left(\mathrm{PPh}_{3}\right)_{2}$ with alkylating agents gives the known $\sigma$ - and $\eta$ organocobalt $(\mathrm{I})$ derivatives, and reactions with $\mathrm{TlClO}_{4}$ in the presence of various amounts of different mono- and bi-dentate phosphines give the cationic tricarbonyl $\left[\mathrm{Co}(\mathrm{CO})_{3}\left(\mathrm{PPh}_{3}\right)_{2}\right]^{+}$, dicarbonyl $\left[\mathrm{Co}(\mathrm{CO})_{2}\left(\mathrm{PMePh}_{2}\right)_{3}\right]^{+}$and monocarbonyl $\left[\mathrm{Co}(\mathrm{CO}) \mathrm{L}_{4}\right]^{+}$complexes $\left(\mathrm{L}_{4}=4 \mathrm{P}(\mathrm{OMe})_{3}, 2 \mathrm{dppe}\right.$ and $\left.2 \mathrm{dppm}\right)$. The dppm complex crystallizes in the monoclinic space group $P 2_{1} / c$ with $a 17.895(6), b 10.751(2), c$ 24.687(4) $\AA, \beta 98.92(1)^{\circ}$, and $D_{\text {calc }} 1.35 \mathrm{~g} \mathrm{~cm}^{-3}$ for $Z=4$. A final $R$ value of 0.077 $\left(R_{\mathrm{w}}=0.061\right.$ ), based on 2656 observed reflections, was obtained. The cobalt atom exhibits a distorted trigonal bipyramidal geometry. The perchlorate anion is severely disordered or freely rotating.

\section{Introduction}

We previously described the preparation of dimeric or polymeric halotriphenylphosphinecobalt $(\mathrm{I})\left[\mathrm{XCo}\left(\mathrm{PPh}_{3}\right)_{2}\right]_{n}$ and $\left[\mathrm{XCo}\left(\mathrm{PPh}_{3}\right)\right]_{n}$ compounds and the intermediate formation of unstable $\left[\mathrm{X}_{2} \mathrm{Co}\left(\mathrm{PPh}_{3}\right)_{2}\right]^{-}$by reduction of cobalt(II) halides with $\mathrm{NaBH}_{4}$ [1].

We now describe the reactions of these cobalt(I) derivatives with $\mathrm{CO}$ and the behaviour of $\mathrm{ClCo}(\mathrm{CO})_{2}\left(\mathrm{PPh}_{3}\right)_{2}$ in several substitution reactions; the latter have given several known carbonylcobalt(I) compounds and also the new complex $\left[\mathrm{Co}(\mathrm{CO})(\mathrm{dppm})_{2}\right] \mathrm{ClO}_{4}$, which has been characterized by X-ray diffraction. 


\section{Results and discussion}

We previously reported that anionic cobalt(I) species of the type $\left[\mathrm{X}_{2} \mathrm{Co}\left(\mathrm{PPh}_{3}\right)_{2}\right]^{-}$, formed by reducing cobalt(II) halides with $\mathrm{NaBH}_{4}$ in the presence of appropriate molar ratios of $\mathrm{PPh}_{3}$ and $\mathrm{PR}_{4} \mathrm{X}$, could not be isolated because they were unstable in solution, and deproportionated readily to give cobalt( 0$)$ and cobalt(II) derivatives, which were recovered as the anionic $\left[\mathrm{X}_{3} \mathrm{Co}\left(\mathrm{PPh}_{3}\right)\right]^{--}$complexes [1]. However, when the same reaction was carried out in the presence of $\mathrm{CO}$ the initially blue suspensions turned green, and work up yielded green-yellow crystals of $\left[\mathrm{X}_{2} \mathrm{Co}(\mathrm{CO})_{2}\left(\mathrm{PPh}_{3}\right)\right]^{-}$ $\mathrm{PR}_{4}{ }^{+}\left(\mathrm{X}=\mathrm{Cl}, \mathrm{PR}_{4}=\mathrm{PBzPh}_{3}(\mathrm{I}) ; \mathrm{X}=\mathrm{Br}, \mathrm{PR}_{4}=\mathrm{PEtPh}_{3}\right.$ (II). When $\mathrm{X}=\mathrm{I}$, the anionic complex is extensively dissociated, and only the neutral complex formed by addition of $\mathrm{PPh}_{3}$ was isolated (see eq. 1).

$$
\mathrm{I}_{2} \mathrm{Co}(\mathrm{CO})_{2}\left(\mathrm{PPh}_{3}\right)^{-} \stackrel{\mathrm{PPh}_{3}}{\longrightarrow} \mathrm{I}^{-}+\mathrm{ICo}(\mathrm{CO})_{2}\left(\mathrm{PPh}_{3}\right)_{2}
$$

Complexes I and II are diamagnetic as solids at room temperature and give conducting solutions in acetone with conductivities of 92 (I) and 75 (II) $\Omega^{-1} \mathrm{~cm}^{2}$ $\mathrm{mol}^{-1}$, consistent with their formulation as $1 / 1$ electrolytes [2]. The IR spectra in $\mathrm{KBr}$ pellets show absorptions due to the phosphine ligand and the phosphonium cation [3], along with two $\nu(\mathrm{CO})$ stretching vibrations at 1970,"1900 and 1975, 1910 $\mathrm{cm}^{-1}$, respectively, for I and II. Both complexes are stable as solids under inert atmosphere but IR studies in solution are precluded by their low stabilities. No definitive structural assignment could be made on the basis of these experimental data.

Suspensions of dimeric or polymeric $\left[\mathrm{XCo}\left(\mathrm{PPh}_{3}\right)_{2}\right]_{n}[1]$ in ethanol on treatment with $\mathrm{CO}$ at room pressure gave yellow-orange suspensions from which the previously reported complexes $\mathrm{XCo}(\mathrm{CO})_{2}\left(\mathrm{PPh}_{3}\right)_{2}(\mathrm{X}=\mathrm{Cl}, \mathrm{Br}, \mathrm{I})$ [4-6] were isolated. In these reactions the halogen bridge is cleaved by $\mathrm{CO}$ with increase in the coordination number and formation of the pentacoordinate complexes. The same products are obtained by reaction with $\mathrm{CO}$ of polymeric $\left[\mathrm{XCo}\left(\mathrm{PPh}_{3}\right)\right]_{n}[1]$; the expected tricarbonyl derivatives are unstable [7] and readily converted into the dicarbonyl complexes, probably by the dissociated free phosphine. The structures of these dicarbonyl complexes were assigned on the basis of IR and ${ }^{31} \mathrm{P}$ NMR studies [8].

The behaviour of $\mathrm{ClCo}(\mathrm{CO})_{2}\left(\mathrm{PPh}_{3}\right)_{2}$ in different types of substitution reactions was studied. The chloro complex reacts with $\mathrm{Mg}\left(\mathrm{C}_{6} \mathrm{~F}_{5}\right) \mathrm{Br}$ to give the $\sigma$-organocarbonyl derivative $\left(\mathrm{C}_{6} \mathrm{~F}_{5}\right) \mathrm{Co}(\mathrm{CO})_{2}\left(\mathrm{PPh}_{3}\right)_{2}$, which was previously obtained by a different route [8]. In a similar reaction with $\mathrm{Mg}\left(\mathrm{C}_{3} \mathrm{H}_{5}\right) \mathrm{Br}$ at $-20^{\circ} \mathrm{C}$ an orange red solution was obtained, and work-up gave yellow crystals which were identified as $\left(\eta^{3}-\mathrm{C}_{3} \mathrm{H}_{5}\right) \mathrm{Co}(\mathrm{CO})_{2}\left(\mathrm{PPh}_{3}\right)$, which was also obtained previously by other methods, and whose X-ray structure is known [9]. In this reaction coordination of the $\eta^{3}-\mathrm{C}_{3} \mathrm{H}_{5}$ group involves preferential elimination of $\mathrm{PPh}_{3}$.

A similar reaction with $\mathrm{Na}\left(\mathrm{C}_{5} \mathrm{H}_{5}\right)$ in $\mathrm{THF}$ gave a red solution, and $\left(\eta^{5}-\mathrm{C}_{5} \mathrm{H}_{5}\right)$ $\mathrm{Co}(\mathrm{CO})_{2}[10]$ was identified by IR and ${ }^{1} \mathrm{H}$ NMR spectroscopy in the condensed volatile fraction obtained by evaporation. The resulting red solid residue contained $\left(\eta^{5}-\mathrm{C}_{5} \mathrm{H}_{5}\right) \mathrm{Co}(\mathrm{CO})\left(\mathrm{PPh}_{3}\right)$ [11] which was isolated by chromatography after extraction with pentane. This solid does not contain $\left(\eta^{5}-\mathrm{C}_{5} \mathrm{H}_{5}\right) \mathrm{Co}\left(\mathrm{PPh}_{3}\right)_{2}$ and so coordination of the $\eta^{5}-\mathrm{C}_{5} \mathrm{H}_{5}$ group again involves preferential elimination of $\mathrm{PPh}_{3}$ in the first step, although a carbonyl group can be displaced in a second step. 
TABLE 1

ANALYSES AND PHYSICAL PROPERTIES OF NEW COBALT(I) COMPLEXES

\begin{tabular}{|c|c|c|c|c|c|}
\hline \multirow[t]{2}{*}{$\overline{\text { Complex }}$} & \multicolumn{3}{|c|}{$\begin{array}{l}\text { Analysis } \\
\text { (Found (calcd.)(\%)) }\end{array}$} & \multirow[t]{2}{*}{ Colour } & \multirow[t]{2}{*}{$\begin{array}{l}\Lambda \text { (acetone) }\left(10^{-4} M\right) \\
\left(\Omega^{-1} \mathrm{~cm}^{2} \mathrm{~mol}^{-1}\right)\end{array}$} \\
\hline & $\mathrm{C}$ & $\mathrm{H}$ & $\mathrm{Cl}$ & & \\
\hline$\left(\mathrm{PBzPh}_{3}\right)\left[\mathrm{Cl}_{2} \mathrm{Co}(\mathrm{CO})_{2}\left(\mathrm{PPh}_{3}\right)\right]$ & $\begin{array}{c}67.05 \\
(67.42)\end{array}$ & $\begin{array}{c}5.17 \\
(4.65)\end{array}$ & $\begin{array}{c}8.82 \\
(8.80)\end{array}$ & green-yellow & 92.3 \\
\hline$\left(\mathrm{PEtPh}_{3}\right)\left[\mathrm{Br}_{2} \mathrm{Co}(\mathrm{CO})_{2}\left(\mathrm{PPh}_{3}\right)\right]$ & $\begin{array}{c}57.54 \\
(57.99)\end{array}$ & $\begin{array}{c}4.61 \\
(4.27)\end{array}$ & - & green-yellow & 75.1 \\
\hline$\left[\mathrm{Co}(\mathrm{CO})\left(\mathrm{Ph}_{2} \mathrm{PCH}_{2} \mathrm{PPh}_{2}\right)_{2}\right]\left(\mathrm{ClO}_{4}\right)$ & $\begin{array}{c}64.06 \\
(64.11)\end{array}$ & $\begin{array}{c}4.92 \\
(4.65)\end{array}$ & - & orange & 127.0 \\
\hline
\end{tabular}

The preferential displacement of $\mathrm{PPh}_{3}$ is also observed when $\mathrm{ClCo}(\mathrm{CO})_{2}\left(\mathrm{PPh}_{3}\right)_{2}$ reacts with $\mathrm{P}(\mathrm{OMe})_{3}$. The reaction goes through the intermediate $\mathrm{ClCo}(\mathrm{CO})_{2}-$ $\left[\mathrm{P}(\mathrm{OMe})_{3}\right]_{2}$, (compare the similar behaviour when $\left.\mathrm{L}=\mathrm{P}(\mathrm{OPh})_{3}[7,12]\right)$ to give $\mathrm{Co}(\mathrm{CO})\left[\mathrm{P}(\mathrm{OMe})_{3}\right]_{4}{ }^{+}$when an excess of the ligand is present. After addition of $\mathrm{NaBPh}_{4}$, this complex was isolated as the $\mathrm{BPh}_{4}$ salt, previously obtained from $\mathrm{Co}_{2}(\mathrm{CO})_{8}[13]$.

Different behaviour is observed when ligands are added after prior elimination of halide by use of a thallous or silver salt. The reaction of $\mathrm{ClCO}(\mathrm{CO})_{2}\left(\mathrm{PPh}_{3}\right)_{2}$ with $\mathrm{AgClO}_{4}$ has been reported [14] to give neutral $\mathrm{Co}\left(\mathrm{OClO}_{3}\right)(\mathrm{CO})_{2}\left(\mathrm{PPh}_{3}\right)_{2}$. We did not isolate this compound after addition of $\mathrm{AgClO}_{4}$ or $\mathrm{TlClO}_{4}$; instead, after stirring for $2 \mathrm{~h}$ we isolated $\left[\mathrm{Co}(\mathrm{CO})_{3}\left(\mathrm{PPh}_{3}\right)_{2}\right]\left(\mathrm{ClO}_{4}\right)$ as the main product even after addition of an excess of $\mathrm{PPh}_{3}$; the stability of this type of tricarbonyl complex in substitution reactions is well known [15]. However the same reaction with $\mathrm{TlClO}_{4}$ in the presence

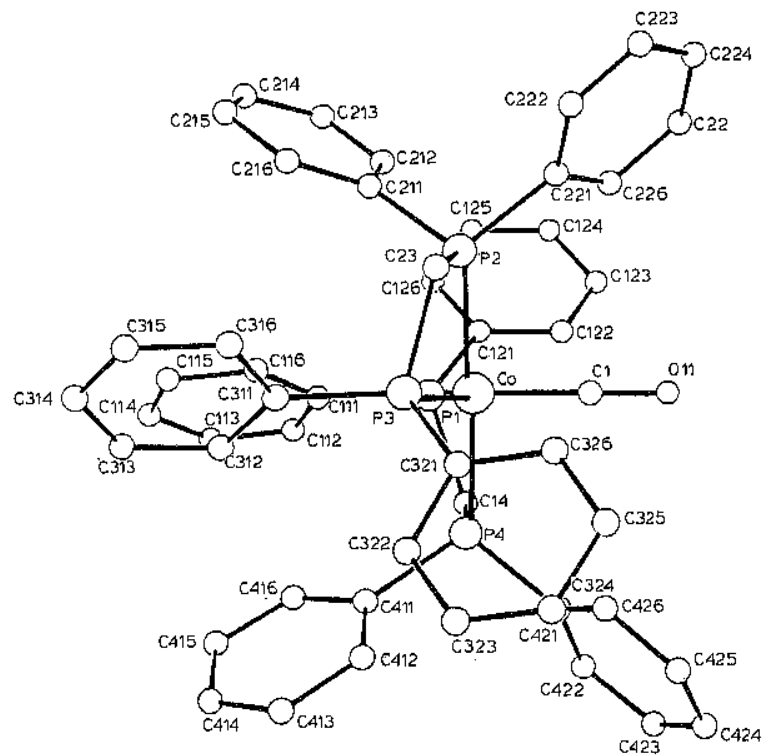

Fig. 1. The molecular structure of the cation $\left[\mathrm{Co}(\mathrm{CO})(\mathrm{dppm})_{2}\right]^{+}$, showing the atom numbering scheme. 
TABLE 2

ATOMIC COORDINATES FOR $\left[\mathrm{CO}(\mathrm{CO})(\mathrm{dppm})_{2}\right]\left(\mathrm{ClO}_{4}\right)$ COORDINATES AND THERMAL PARAMETERS AS $U_{\text {eq }}=\frac{1}{3} \Sigma\left(U_{i j} a_{i}^{\star} a_{j}^{\star} a_{i} a_{j} \cos \left(a_{i} a_{j}\right)\right) \times 10^{4}$

\begin{tabular}{|c|c|c|c|c|}
\hline Atom & $x / a$ & $y / b$ & $z / c$ & $U_{\text {eq }}$ \\
\hline $\mathrm{Co}$ & $0.20492(8)$ & $0.23657(16)$ & $0.57643(6)$ & $328(6)$ \\
\hline $\mathrm{P}(1)$ & $0.24241(21)$ & $0.03959(34)$ & $0.58901(15)$ & $404(14)$ \\
\hline $\mathrm{P}(2)$ & $0.17675(18)$ & $0.28871(31)$ & $0.65713(13)$ & $371(12)$ \\
\hline $\mathrm{P}(3)$ & $0.25664(20)$ & $0.42280(32)$ & $0.59553(15)$ & $383(14)$ \\
\hline$P(4)$ & $0.25048(20)$ & $0.18212(30)$ & $0.50157(14)$ & $387(12)$ \\
\hline $\mathrm{Cl}$ & $0.30291(29)$ & $0.65754(49)$ & $0.82342(23)$ & $943(23)$ \\
\hline$O(1)$ & $0.32933(140)$ & $0.60054(243)$ & $0.87732(116)$ & $1657(99)$ \\
\hline$O(2)$ & $0.32419(179)$ & $0.77482(338)$ & $0.84024(151)$ & $2139(138)$ \\
\hline $\mathrm{O}(3)$ & $0.22611(194)$ & $0.64363(299)$ & $0.82220(141)$ & $1684(122)$ \\
\hline$O(4)$ & $0.33899(209)$ & $0.58712(362)$ & $0.78724(160)$ & $113(106)$ \\
\hline$O(11)$ & $0.04928(52)$ & $0.25583(144)$ & $0.52109(41)$ & $1057(56)$ \\
\hline$C(1)$ & $0.11102(76)$ & $0.24675(139)$ & $0.54352(57)$ & $591(56)$ \\
\hline $\mathrm{C}(23)$ & $0.19986(66)$ & $0.45251(124)$ & $0.65110(50)$ & $451(52)$ \\
\hline $\mathrm{C}(14)$ & $0.24408(74)$ & $0.01584(105)$ & $0.51542(48)$ & $390(50)$ \\
\hline$C(111)$ & $0.33665(82)$ & $-0.00240(127)$ & $0.62075(55)$ & $501(58)$ \\
\hline $\mathrm{C}(112)$ & $0.37224(93)$ & $-0.11061(129)$ & $0,61060(61)$ & $627(67)$ \\
\hline $\mathrm{C}(113)$ & $0.44833(100)$ & $-0.12910(176)$ & $0.63113(85)$ & $875(86)$ \\
\hline$C(114)$ & $0.48866(91)$ & $-0.04131(214)$ & $0.66297(77)$ & $875(85)$ \\
\hline $\mathrm{C}(115)$ & $0.45463(86)$ & $0.06785(157)$ & $0.67510(70)$ & $726(70)$ \\
\hline$C(116)$ & $0.37746(85)$ & $0.08808(144)$ & $0.65361(53)$ & $625(63)$ \\
\hline $\mathrm{C}(121)$ & $0.18131(77)$ & $-0.08324(120)$ & $0.61171(57)$ & $490(56)$ \\
\hline$C(122)$ & $0.10839(82)$ & $-0.09422(127)$ & $0.58534(58)$ & $567(58)$ \\
\hline$C(123)$ & $0.05962(81)$ & $-0.17748(150)$ & $0.60463(69)$ & $692(66)$ \\
\hline $\mathrm{C}(124)$ & $0.08386(101)$ & $-0.24688(162)$ & $0.65188(69)$ & $818(76)$ \\
\hline$C(125)$ & $0.15543(95)$ & $-0.23439(149)$ & $0.67711(62)$ & $663(63)$ \\
\hline $\mathrm{C}(126)$ & $0.20541(84)$ & $-0.15337(133)$ & $0.65715(56)$ & $503(57)$ \\
\hline$C(211)$ & $0.22999(70)$ & $0.22474(128)$ & $0.71913(48)$ & $431(45)$ \\
\hline $\mathrm{C}(212)$ & $0.21301(79)$ & $0.10448(138)$ & $0.73403(58)$ & $528(61)$ \\
\hline$C(213)$ & $0.24972(114)$ & $0.05182(148)$ & $0.77973(77)$ & $795(81)$ \\
\hline$C(214)$ & $0.30719(115)$ & $0.11481(254)$ & $0.81110(71)$ & $913(97)$ \\
\hline$C(215)$ & $0.32377(95)$ & $0.23237(231)$ & $0.80010(66)$ & $852(81)$ \\
\hline$C(216)$ & $0.28650(85)$ & $0.29360(146)$ & $0.75278(61)$ & $643(64)$ \\
\hline$C(221)$ & $0.07837(61)$ & $0.28453(136)$ & $0.67079(47)$ & $468(48)$ \\
\hline$C(222)$ & $0.05169(75)$ & $0.38073(140)$ & $0.70052(55)$ & $579(58)$ \\
\hline$C(223)$ & $-0.01804(118)$ & $0.37957(209)$ & $0.71268(73)$ & $957(95)$ \\
\hline$C(224)$ & $-0.06393(99)$ & $0.28068(289)$ & $0.69555(87)$ & $1115(112)$ \\
\hline$C(225)$ & $-0.04065(87)$ & $0.18004(189)$ & $0.66568(82)$ & $937(85)$ \\
\hline$C(226)$ & $0.03366(75)$ & $0.18492(141)$ & $0.65253(58)$ & $637(61)$ \\
\hline$C(311)$ & $0.35289(69)$ & $0.45623(121)$ & $0.62689(44)$ & $392(45)$ \\
\hline$C(312)$ & $0.41143(81)$ & $0.38001(137)$ & $0.61695(57)$ & $586(59)$ \\
\hline$C(313)$ & $0.48616(79)$ & $0.39807(176)$ & $0.64410(66)$ & $741(70)$ \\
\hline$C(314)$ & $0.50011(108)$ & $0.50037(202)$ & $0.67870(77)$ & $869(89)$ \\
\hline$C(315)$ & $0.44477(108)$ & $0.57476(173)$ & $0.68840(64)$ & $769(76)$ \\
\hline$C(316)$ & $0.36896(90)$ & $0.55737(123)$ & $0.66351(59)$ & $622(62)$ \\
\hline$C(321)$ & $0.23486(78)$ & $0.54792(119)$ & $0.54530(49)$ & $480(52)$ \\
\hline$C(322)$ & $0.29170(76)$ & $0.58949(125)$ & $0.51610(57)$ & $541(53)$ \\
\hline$C(323)$ & $0.27301(100)$ & $0.67219(140)$ & $0.47208(62)$ & $670(67)$ \\
\hline$C(324)$ & $0.20306(94)$ & $0.70833(146)$ & $0.45511(68)$ & $729(68)$ \\
\hline$C(32,5)$ & $0.14336(94)$ & $0.66669(144)$ & $0.48123(69)$ & $685(68)$ \\
\hline$C(326)$ & $0.16081(84)$ & $0.58512(147)$ & $0.52714(71)$ & $717(69)$ \\
\hline$C(411)$ & $0.34846(66)$ & $0.21507(119)$ & $0.49309(47)$ & $374(45)$ \\
\hline$C(412)$ & $0.36185(78)$ & $0.32522(141)$ & $0.46719(60)$ & $584(60)$ \\
\hline
\end{tabular}


TABLE 2 (continued)

\begin{tabular}{lllll}
\hline Atom & $x / a$ & $y / b$ & $z / c$ & $U_{\text {eq }}$ \\
\hline $\mathrm{C}(413)$ & $0.43614(115)$ & $0.36361(167)$ & $0.46236(75)$ & $831(83)$ \\
$\mathrm{C}(414)$ & $0.49426(95)$ & $0.28824(222)$ & $0.48520(80)$ & $899(87)$ \\
$\mathrm{C}(415)$ & $0.48263(90)$ & $0.17908(198)$ & $0.51257(80)$ & $922(85)$ \\
$\mathrm{C}(416)$ & $0.40931(80)$ & $0.13833(149)$ & $0.51472(63)$ & $647(65)$ \\
$\mathrm{C}(421)$ & $0.19681(70)$ & $0.21432(134)$ & $0.43442(50)$ & $453(50)$ \\
$\mathrm{C}(422)$ & $0.19275(87)$ & $0.13020(138)$ & $0.39182(67)$ & $639(64)$ \\
$\mathrm{C}(423)$ & $0.15171(83)$ & $0.15838(167)$ & $0.34106(56)$ & $597(67)$ \\
$\mathrm{C}(424)$ & $0.11306(83)$ & $0.26805(200)$ & $0.33243(63)$ & $762(71)$ \\
$\mathrm{C}(425)$ & $0.12120(83)$ & $0.35573(157)$ & $0.37456(77)$ & $684(70)$ \\
$\mathrm{C}(426)$ & $0.16071(89)$ & $0.32556(141)$ & $0.42594(63)$ & $584(62)$ \\
$\mathrm{O}(22)$ & $0.34452(298)$ & $0.55331(514)$ & $0.82567(228)$ & $1772(312)$ \\
$\mathrm{O}(21)$ & $0.25897(193)$ & $0.77223(345)$ & $0.83328(143)$ & $1197(184)$ \\
$\mathrm{O}(23)$ & $0.23653(194)$ & $0.59093(332)$ & $0.78955(148)$ & $1418(203)$ \\
$\mathrm{O}(24)$ & $0.33932(188)$ & $0.70093(331)$ & $0.78101(157)$ & $3022(262)$ \\
\hline
\end{tabular}

of $\mathrm{PMePh}_{2}$ gave the cationic dicarbonyl complex $\left[\mathrm{Co}(\mathrm{CO})_{2}\left(\mathrm{PMePh}_{2}\right)_{3}\right]\left(\mathrm{ClO}_{4}\right)$, previously obtained by a different method [16]. Similar reactions with bidentate phosphines give monocarbonyl cationic complexes $\left[\mathrm{Co}(\mathrm{CO})(\mathrm{L}-\mathrm{L})_{2}\right]\left(\mathrm{ClO}_{4}\right)$, with $\mathrm{L}-\mathrm{L}=$ dppe [17] or $\mathrm{L}-\mathrm{L}=$ dppm.

The dppm complex is a diamagnetic solid soluble in $\mathrm{CH}_{2} \mathrm{Cl}_{2}$ and acetone to give conducting solutions with a conductivity of $127 \Omega^{-1} \mathrm{~cm}^{2} \mathrm{~mol}^{-1}$. It shows the $\nu$ (CO) stretching vibration at $1960 \mathrm{~cm}^{-1}\left(\mathrm{CH}_{2} \mathrm{Cl}_{2}\right)$. The ${ }^{31} \mathrm{P} \mathrm{NMR}$ spectrum shows a singlet at $\delta-6.64 \mathrm{ppm}$ (acetone- $d_{6}$ ) between $-80^{\circ} \mathrm{C}$ and room temperature. The ${ }^{1} \mathrm{H}$ NMR spectrum shows a triplet at $\delta 4.05 \mathrm{ppm}$ due to the $\mathrm{CH}_{2}$ protons and a multiplet at $\delta 6.86 \mathrm{ppm}$ due to the Ph protons. These data imply fluxional behaviour [18], and it was therefore of interest to determine the structure of the complex by $\mathrm{X}$-ray diffraction.

The analyses and physical properties of some of the new cobalt(I) complexes are given in Table 1.

\section{$X$-Ray structure of $\left[\mathrm{Co}(\mathrm{CO})(\mathrm{dppm})_{2}\right]\left(\mathrm{ClO}_{4}\right)$}

An ORTEP drawing of the title compound based on the X-ray structural analysis, is shown in Fig. 1. Atomic parameters for the non hydrogen atoms are listed in Table 2. Important bond distances and bond angles are listed in Table 3. Equations for selected least squares mean planes are given in Table 4. Tables of thermal parameters and structure factors are available from the authors.

The geometry about the cobalt atom aproximates to a trigonal bipyramid, but because of the rigidity of the ligand it is very distorted, and an intermediate between the usual coordination geometries for 18 -electron cobalt(I) complexes, i.e. square pyramidal or trigonal bipyramidal. The $\mathrm{Co}, \mathrm{P}(1), \mathrm{P}(3), \mathrm{C}(1)$, and $\mathrm{O}(11)$ atoms define the equatorial plane, and the $\mathrm{P}(2)$ and $\mathrm{P}(4)$ atoms lie in axial positions, practically equidistant from this plane $\mathrm{P}(2) 2.115(3) \AA$ and $\mathrm{P}(4) 2.123(3) \AA$. The angle $\mathrm{P}(2)-\mathrm{Co}-\mathrm{P}(4)$ between the two axial phosphorus atoms is $171.61(5)^{\circ}$; the deviation from linearity is due to the strain in these chelate complexes.

The two $\mathrm{P}$ atoms of each dppm ligand occupy one axial and one equatorial site [19]. The angles of this chelate system are similar to those previously reported [20]. 
TABLE 3

INTERATOMIC DISTANCES $(\AA)$ AND ANGLES $\left({ }^{\circ}\right)$ WITH E.S.D.'S IN PARENTHESES FOR $\left[\mathrm{Co}(\mathrm{CO})(\mathrm{dppm})_{2}\right] \mathrm{ClO}_{4}$

\begin{tabular}{|c|c|c|c|}
\hline $\mathrm{Co}-\mathrm{P}(1)$ & $2.228(4)$ & $\mathrm{C}(221)-\mathrm{C}(226)$ & $1.370(19)$ \\
\hline $\mathrm{Co}-\mathrm{P}(2)$ & $2.202(4)$ & $C(222)-C(223)$ & $1.328(25)$ \\
\hline $\mathrm{Co}-\mathrm{P}(3)$ & $2.225(4)$ & $C(223)-C(224)$ & $1.369(34)$ \\
\hline Co-P(4) & $2.211(4)$ & $C(224)-C(225)$ & $1.408(34)$ \\
\hline $\mathrm{Co}-\mathrm{C}(1)$ & $1.752(13)$ & $C(225)-C(226)$ & $1.418(22)$ \\
\hline$P(1)-C(14)$ & $1.839(13)$ & $\mathrm{C}(311)-\mathrm{C}(312)$ & $1.382(19)$ \\
\hline$P(1)-C(111)$ & $1.803(14)$ & $C(311)-C(316)$ & $1.415(18)$ \\
\hline$P(1)-C(121)$ & $1.855(14)$ & $C(312)-C(313)$ & $1.413(19)$ \\
\hline$P(2)-C(23)$ & $1.820(13)$ & $C(313)-C(314)$ & $1.391(27)$ \\
\hline$P(2)-C(211)$ & $1.808(12)$ & $C(314)-C(315)$ & $1.323(28)$ \\
\hline $\mathrm{P}(2)-\mathrm{C}(221)$ & $1.843(12)$ & $C(315)-C(316)$ & $1.412(23)$ \\
\hline $\mathrm{P}(3)-\mathrm{C}(23)$ & $1.857(13)$ & $\mathrm{C}(321)-\mathrm{C}(322)$ & $1.407(20)$ \\
\hline$P(3)-C(311)$ & $1.811(12)$ & $\mathrm{C}(321)-\mathrm{C}(326)$ & $1.390(19)$ \\
\hline$P(3)-C(321)$ & $1.830(13)$ & $C(322)-C(323)$ & $1.403(20)$ \\
\hline$P(4)-C(14)$ & $1.827(12)$ & $C(323)-C(324)$ & $1.316(23)$ \\
\hline$P(4)-C(411)$ & $1.832(13)$ & $C(324)-C(325)$ & $1.404(25)$ \\
\hline$P(4)-C(421)$ & $1.816(12)$ & $\mathrm{C}(325)-\mathrm{C}(326)$ & $1.428(23)$ \\
\hline $\mathrm{C}(1)-\mathrm{O}(11)$ & $1.161(15)$ & $\mathrm{C}(411)-\mathrm{C}(412)$ & $1.384(19)$ \\
\hline $\mathrm{C}(111)-\mathrm{C}(112)$ & $1.368(20)$ & $C(411)-C(416)$ & $1.404(18)$ \\
\hline $\mathrm{C}(111)-\mathrm{C}(118)$ & $1.398(19)$ & $C(412)-C(413)$ & $1.415(25)$ \\
\hline$C(112)-C(113)$ & $1.392(23)$ & $C(413)-C(414)$ & $1.369(26)$ \\
\hline $\mathrm{C}(113)-\mathrm{C}(114)$ & $1.361(27)$ & $C(414)-C(415)$ & $1.386(31)$ \\
\hline$C(114)-C(115)$ & $1.376(27)$ & $C(415)-C(416)$ & $1.392(22)$ \\
\hline$C(115)-C(116)$ & $1.417(20)$ & $C(421)-C(422)$ & $1.380(20)$ \\
\hline$C(121)-C(122)$ & $1.370(19)$ & $C(421)-C(426)$ & $1.360(20)$ \\
\hline$C(121)-C(126)$ & $1.365(19)$ & $C(422)-C(423)$ & $1.384(20)$ \\
\hline$C(122)-C(123)$ & $1.385(22)$ & $C(423)-C(424)$ & $1.367(26)$ \\
\hline $\mathrm{C}(123)-\mathrm{C}(124)$ & $1.396(23)$ & $\mathrm{C}(424)-\mathrm{C}(425)$ & $1.394(25)$ \\
\hline$C(124)-C(125)$ & $1.341(23)$ & $C(429)-C(426)$ & $1.391(22)$ \\
\hline$C(125)-C(126)$ & $1.392(23)$ & $\mathrm{Cl}-\mathrm{O}(1)$ & $1.474(27)$ \\
\hline$C(211)-C(212)$ & $1.391(20)$ & $\mathrm{Cl}-\mathrm{O}(2)$ & $1.363(36)$ \\
\hline$C(211)-C(216)$ & $1.414(19)$ & $\mathrm{Cl}-\mathrm{O}(3)$ & $1.378(35)$ \\
\hline$C(212)-C(213)$ & $1.340(22)$ & $\mathrm{Cl}-\mathrm{O}(4)$ & $1.402(41)$ \\
\hline$C(213)-C(214)$ & $1.367(27)$ & $\mathrm{Cl}-\mathrm{O}(21)$ & $1.502(27)$ \\
\hline$C(214)-C(215)$ & $1.336(36)$ & $\mathrm{Cl}-\mathrm{O}(22)$ & $1.341(55)$ \\
\hline$C(215)-C(216)$ & $1.414(26)$ & $\mathrm{Cl}-\mathrm{O}(23)$ & $1.521(33)$ \\
\hline$C(221)-C(222)$ & $1.395(20)$ & $\mathrm{Cl}-\mathrm{O}(24)$ & $1.396(39)$ \\
\hline $\mathrm{P}(4)-\mathrm{Co}-\mathrm{C}(1)$ & $94.6(4)$ & $\mathrm{P}(3)-\mathrm{Co}-\mathrm{C}(1)$ & $112.3(5)$ \\
\hline $\mathrm{P}(3)-\mathrm{Co}-\mathrm{P}(4)$ & $102.7(1)$ & $\mathrm{P}(2)-\mathrm{Co}-\mathrm{C}(1)$ & $93.7(5)$ \\
\hline $\mathrm{P}(2)-\mathrm{Co}-\mathrm{P}(4)$ & $171.6(1)$ & $\mathrm{P}(2)-\mathrm{Co}-\mathrm{P}(3)$ & $74.0(1)$ \\
\hline $\mathrm{P}(1)-\mathrm{Co}-\mathrm{C}(1)$ & $111.7(4)$ & $\mathrm{P}(1)-\mathrm{Co}-\mathrm{P}(4)$ & $73.9(1)$ \\
\hline$P(1)-C o-P(3)$ & $135.9(1)$ & $\mathrm{P}(1)-\mathrm{Co}-\mathrm{P}(2)$ & $102.8(1)$ \\
\hline$P(2)-C(23)-P(3)$ & $92.9(6)$ & $P(1)-C(14)-P(4)$ & $93.4(5)$ \\
\hline $\mathrm{Co}-\mathrm{P}(1)-\mathrm{C}(12 \mathrm{I})$ & $122.6(4)$ & $\mathrm{Co}-\mathrm{P}(1)-\mathrm{C}(111)$ & $122.6(4)$ \\
\hline $\mathrm{Co}-\mathrm{P}(1)-\mathrm{C}(14)$ & $92.5(4)$ & $C(111)-P(1)-C(121)$ & $104.2(6)$ \\
\hline $\mathrm{C}(14)-\mathrm{P}(1)-\mathrm{C}(121)$ & $107.4(5)$ & $C(14)-P(1)-C(111)$ & $103.7(6)$ \\
\hline $\mathrm{Co}-\mathrm{P}(2)-\mathrm{C}(221)$ & $121.2(3)$ & $\mathrm{Co}-\mathrm{P}(2)-\mathrm{C}(211)$ & $120.2(4)$ \\
\hline $\mathrm{Co}-\mathrm{P}(2)-\mathrm{C}(23)$ & $94.9(4)$ & $\mathrm{C}(211)-\mathrm{P}(2)-\mathrm{C}(221)$ & $103.2(5)$ \\
\hline $\mathrm{C}(23)-\mathrm{P}(2)-\mathrm{C}(221)$ & $105.9(5)$ & $\mathrm{C}(23)-\mathrm{P}(2)-\mathrm{C}(211)$ & $109.9(5)$ \\
\hline $\mathrm{Co}-\mathrm{P}(3)-\mathrm{C}(23)$ & $93.1(4)$ & $\mathrm{Co}-\mathrm{P}(3)-\mathrm{C}(311)$ & $127.2(4)$ \\
\hline $\mathrm{Co}-\mathrm{P}(3)-\mathrm{C}(321)$ & $118.6(4)$ & $\mathrm{C}(311)-\mathrm{P}(3)-\mathrm{C}(321)$ & $103.4(5)$ \\
\hline$C(23)-P(3)-C(321)$ & $106.9(6)$ & $C(23)-P(3)-C(311)$ & $104.1(5)$ \\
\hline $\mathrm{Co}-\mathrm{P}(4)-\mathrm{C}(411)$ & $121.8(4)$ & Co-P(4)-C(421) & $120.1(4)$ \\
\hline $\mathrm{Co}-\mathrm{P}(4)-\mathrm{C}(14)$ & $93.4(4)$ & $C(411)-P(4)-C(421)$ & $103.8(8)$ \\
\hline $\mathrm{C}(14)-\mathrm{P}(4)-\mathrm{C}(421)$ & $108.5(5)$ & $\mathrm{C}(14)-\mathrm{P}(4)-\mathrm{C}(11)$ & $107.4(5)$ \\
\hline
\end{tabular}


TABLE 4

$a x+b y+c z=d$ EQUATIONS OF THE PLANES OF SOME PLANAR MOIETIES, AND DEVIATIONS OF THE ATOMS FROM THESE PLANES $(\AA)$

\begin{tabular}{|c|c|c|c|c|c|c|c|c|}
\hline Planes & Atoms & $a$ & $b$ & c & $\bar{d}$ & $\begin{array}{l}\text { Deviation } \\
(\AA)\end{array}$ & $\begin{array}{l}\text { Others } \\
\text { atoms }\end{array}$ & $\begin{array}{l}\text { Deviation } \\
(\AA)\end{array}$ \\
\hline 1 & $\begin{array}{l}\text { Co } \\
P(1) \\
P(3) \\
C(1) \\
O(11)\end{array}$ & 0.4642 & 0.0083 & -0.8856 & -11.7518 & $\begin{array}{c}0.001(1) \\
-0.001(3) \\
-0.001(3) \\
-0.009(13) \\
0.002(9)\end{array}$ & $\begin{array}{l}P(2) \\
P(4)\end{array}$ & $\begin{array}{r}-2.115(3) \\
2.123(3)\end{array}$ \\
\hline 2 & $\begin{array}{l}\mathrm{C}(111) \\
\mathrm{C}(112) \\
\mathrm{C}(113) \\
\mathrm{C}(114) \\
\mathrm{C}(115) \\
\mathrm{C}(116)\end{array}$ & 0.3965 & 0.4184 & -0.8170 & -10.9408 & $\begin{array}{r}0.006(13) \\
-0.009(15) \\
0.007(20) \\
0.005(19) \\
-0.005(16) \\
-0.001(13)\end{array}$ & & \\
\hline 3 & $\begin{array}{l}C(121) \\
C(122) \\
C(123) \\
C(124) \\
C(125) \\
C(126)\end{array}$ & 0.3804 & -0.7264 & -0.572 .2 & -7.5410 & $\begin{array}{r}-0.002(13) \\
-0.006(14) \\
0.014(16) \\
-0.007(17) \\
-0.006(15) \\
0.008(14)\end{array}$ & & \\
\hline 4 & $\begin{array}{l}C(211) \\
C(212) \\
C(213) \\
C(214) \\
C(215) \\
C(216)\end{array}$ & 0.7707 & -0.3546 & -0.5293 & -9.0999 & $\begin{array}{r}0.009(12) \\
-0.003(14) \\
-0.020(19) \\
0.034(20) \\
-0.010(18) \\
-0.007(15)\end{array}$ & & \\
\hline 5 & $\begin{array}{l}C(221) \\
C(222) \\
C(223) \\
C(224) \\
C(225) \\
C(226)\end{array}$ & -0.2038 & 0.4831 & -0.8514 & -12.2112 & $\begin{array}{r}-0.003(12) \\
-0.000(13) \\
0.004(19) \\
0.001(23) \\
-0.009(19) \\
0.007(14)\end{array}$ & & \\
\hline 6 & $\begin{array}{l}C(311) \\
C(312) \\
C(313) \\
C(314) \\
C(315) \\
C(316)\end{array}$ & 更 & 0.5836 & -0.7597 & -7.6270 & $\begin{array}{r}-0.003(11) \\
0.014(14) \\
-0.021(17) \\
0.012(19) \\
0.005(16) \\
-0.005(14)\end{array}$ & & \\
\hline 7 & $\begin{array}{l}C(321) \\
C(322) \\
C(323) \\
C(324) \\
C(325) \\
C(326)\end{array}$ & -0.0477 & -0.7867 & -0.6154 & -12.9067 & $\begin{array}{r}-0.013(12) \\
0.019(13) \\
-0.011(15) \\
-0.005(16) \\
0.010(16) \\
0.004(16)\end{array}$ & & \\
\hline
\end{tabular}


TABLE 4 (continued)

\begin{tabular}{|c|c|c|c|c|c|c|c|c|}
\hline Planes & Atoms & $a$ & $b$ & $c$ & $d$ & $\begin{array}{l}\text { Deviation } \\
(\AA)\end{array}$ & $\begin{array}{l}\text { Others } \\
\text { atoms }\end{array}$ & $\begin{array}{l}\text { Deviation } \\
(\AA)\end{array}$ \\
\hline 8 & $\begin{array}{l}C(411) \\
C(412) \\
C(413) \\
C(414) \\
C(415) \\
C(416)\end{array}$ & 0.0858 & -0.4867 & -0.8693 & -11.1999 & $\begin{array}{r}-0.006(11) \\
-0.004(14) \\
0.012(18) \\
0.004(20) \\
-0.031(19) \\
0.023(15)\end{array}$ & & \\
\hline 9 & $\begin{array}{l}C(421) \\
C(422) \\
C(423) \\
C(424) \\
C(425) \\
C(426)\end{array}$ & 0.8725 & 0.3964 & -0.2856 & -0.4833 & $\begin{array}{r}-0.007(12) \\
0.009(15) \\
0.012(15) \\
-0.035(15) \\
0.031(15) \\
-0.009(15)\end{array}$ & & \\
\hline \multicolumn{9}{|c|}{ Dihedral angles between planes } \\
\hline $\begin{array}{l}2-3 \\
6-7\end{array}$ & & \multicolumn{2}{|c|}{$\begin{array}{l}71.6(4) \\
90.3(4)\end{array}$} & \multicolumn{2}{|r|}{$4-5$} & \multicolumn{2}{|c|}{$82.9(5)$} & \\
\hline
\end{tabular}

$\mathrm{P}(1)-\mathrm{Co}-\mathrm{P}(4) \quad 73.9(1)^{\circ} ; \mathrm{P}(3)-\mathrm{Co}-\mathrm{P}(2) \quad 74.1(1)^{\circ}$, and $\mathrm{P}(1)-\mathrm{C}(14)-\mathrm{P}(4) \quad 93.4(5)^{\circ}$; $\mathrm{P}(3)-\mathrm{C}(23)-\mathrm{P}(2) 92.9(6)^{\circ}$. The carbonyl group does not significantly deviate from linearity $\mathrm{Co}-\mathrm{C}(1)-\mathrm{O}(11) 178.4(1.3)^{\circ}$. The angles in the equatorial plane are $\mathrm{C}(1)-\mathrm{Co}-\mathrm{P}(1) 111.7(5)^{\circ} ; \mathrm{C}(1)-\mathrm{Co}-\mathrm{P}(3) 112.2(5)^{\circ} ; \mathrm{P}(1)-\mathrm{Co}-\mathrm{P}(3) 135.9(1)^{\circ}$; they are significantly different from the theoretical angles for trigonal coordination. The angles $\mathrm{P}(2)-\mathrm{Co}-\mathrm{C}(1) 93.7(4)^{\circ}$ and $\mathrm{P}(4)-\mathrm{Co}-\mathrm{C}(1) 94.6(4)^{\circ}$ are in good agreement with the proposed coordination.

The Co-P distances have the expected values, $\mathrm{Co}-\mathrm{P}(1)$ 2.228(4), $\mathrm{Co}-\mathrm{P}(2) 2.202(4)$, $\mathrm{Co}-\mathrm{P}(3) 2.225(4), \mathrm{Co}-\mathrm{P}(4) 2.211(4) \AA[21,22]$ but are slightly longer than the $\mathrm{Co}-\mathrm{P}$ distances in $\left(\right.$ anti- $\left.\eta^{3}-2,4-\mathrm{Me}_{2} \mathrm{C}_{5} \mathrm{H}_{3}\right) \mathrm{Co}\left[\mathrm{P}\left(\mathrm{CH}_{3}\right)_{3}\right]_{3}$, in which the geometry is square pyramidal [23]. The distortion of the structure produced by the ligand produces small differences in the $\mathrm{Co}-\mathrm{P}$ distances, the largest values are for the equatorial bonds.

The $\mathrm{Co}-\mathrm{C}(1)$ 1.75(1) and $\mathrm{C}(1)-\mathrm{O}(11)$ 1.16(1) $\AA$, and the $\mathrm{P}-\mathrm{C}$ distances, are normal.

The phenyl rings are planar, but the quite high thermal ellipsoids for some $\mathrm{C}$ atoms mean that there is considerable uncertainty in the $\mathrm{C}-\mathrm{C}$ bond lengths.

The perchlorate anion is severely distorted, and is best described as freely rotating. The oxygen atoms were approximated by eight positions of partial occupancy giving $\mathrm{Cl}-\mathrm{O}$ distances varying from $1.34(5)$ to $1.52(3) \AA$, but with meaningless bond angles. Similar behaviour was noted previously $[24,25]$.

\section{Experimental}

All operations were carried out under vacuum or in an inert atmosphere using Schlenk-type glassware. Solvents were dried, distilled and deoxygenated before use. 
The starting cobalt(I) complexes were prepared as previously described [1]. $\mathrm{C}$ and $\mathrm{H}$ analyses were performed with a Perkin-Elmer $240 \mathrm{~B}$ microanalyzer. Conductivities were measured in acetone with a WTW LF-42 conductimeter. IR spectra were recorded in solution or as $\mathrm{KBr}$ mulls between CsI plates using a Perkin-Elmer 599 spectrophotometer. ${ }^{1} \mathrm{H}$ and ${ }^{31} \mathrm{P}$ NMR spectra were recorded on a Varian FT-80 A instrument.

$\left[\mathrm{X}_{2} \mathrm{Co}(\mathrm{CO})_{2}\left(\mathrm{PPh}_{3}\right)\right]\left(\mathrm{PR}_{4}\right)(\mathrm{I}, \mathrm{II})$

$\left[\mathrm{PBzPPh}_{3}\right] \mathrm{Cl}(1.633 \mathrm{~g}, 4.2 \mathrm{mmol})$ and $1.102 \mathrm{~g}(4.2 \mathrm{mmol})$ of $\mathrm{PPh}_{3}$ were added to a solution of $1.00 \mathrm{~g}(4.2 \mathrm{mmol})$ of $\mathrm{CoCl}_{2} \cdot 6 \mathrm{H}_{2} \mathrm{O}$ in ethanol $(40 \mathrm{ml})$ to give a blue suspension. A solution of $0.113 \mathrm{~g}(3 \mathrm{mmol})$ of $\mathrm{NaBH}_{4}$ in ethanol $(20 \mathrm{ml})$ was added dropwise and simultaneous bubbling of $\mathrm{CO}$ through the solution was maintained for $2 \mathrm{~h}$. The resulting yellow-green crystals of I were filtered off, repeatedly washed with ethanol, and dried under vacuum. Yield $60 \%$.

The same procedure using $\mathrm{CoBr}_{2}$ and $\left[\mathrm{PEtPh}_{3}\right] \mathrm{Br}$ gave yellow-green crystals of II. Yield $65 \%$.

The same procedure with $\mathrm{CoI}_{2}$ and $\left[\mathrm{PEtPh}_{3}\right] \mathrm{I}$ gave crystals of $\mathrm{ICO}(\mathrm{CO})_{2}\left(\mathrm{PPh}_{3}\right)_{2}$, after recrystallization from dichloromethane/ethanol. Yield $40 \%$.

Reactions of $\left[\mathrm{XCO}\left(\mathrm{PPh}_{3}\right)_{2}\right]_{n}$ and $\left[\mathrm{XCO}\left(\mathrm{PPh}_{3}\right)\right]_{n}$ with $\mathrm{CO}$

$\mathrm{CO}$ was bubbled at room temperature with stirring for $2 \mathrm{~h}$ through a newly prepared [1] brown suspension of $\left[\mathrm{ClCo}\left(\mathrm{PPh}_{3}\right)_{2}\right]_{n}(3.87 \mathrm{mmol})$ in ethanol $(70 \mathrm{ml})$. The resulting yellow orange solid was filtered off and recrystallized from $\mathrm{CH}_{2} \mathrm{Cl}_{2}$ /ethanol to give $\mathrm{ClCo}(\mathrm{CO})_{2}\left(\mathrm{PPh}_{3}\right)_{2}$ as orange crystals. Yield $90 \%$.

Similar procedures with the bromo and iodo derivatives gave the corresponding bromo- and iodo-dicarbonyl complexes. Yields $85-90 \%$.

In a similar procedure $\mathrm{CO}$ was bubbled at room temperature for $2 \mathrm{~h}$ through brown suspensions of $\left[\mathrm{XCo}\left(\mathrm{PPh}_{3}\right)\right]_{n}(\mathrm{X}=\mathrm{Cl}$ or $\mathrm{I})$. The solid was filtered off and extracted into dichloromethane. Addition of ethanol gave the neutral dicarbonyl complex $\mathrm{XCo}(\mathrm{CO})_{2}\left(\mathrm{PPh}_{3}\right)_{2}$.

Reactions of $\mathrm{XCO}(\mathrm{CO})_{2}\left(\mathrm{PPh}_{3}\right)_{2}$ with $\mathrm{Mg}\left(\mathrm{C}_{6} F_{5}\right) \mathrm{Br}$

A THF solution of $\mathrm{Mg}\left(\mathrm{C}_{6} \mathrm{~F}_{5}\right) \mathrm{Br}(1.68 \mathrm{mmol})$ was added to a suspension of $0.506 \mathrm{~g}$ $(0.75 \mathrm{mmol})$ of $\mathrm{CoCl}(\mathrm{CO})_{2}\left(\mathrm{PPh}_{3}\right)_{2}$ in THF $(40 \mathrm{ml})$. After $1.5 \mathrm{~h}$ stirring at room temperature the orange solution was filtered then evaporated to dryness. The residue was extracted into toluene and addition of hexane gave crystals of $\left(\mathrm{C}_{6} \mathrm{~F}_{5}\right) \mathrm{Co}(\mathrm{CO})_{2-}$ $\left(\mathrm{PPh}_{3}\right)_{2}$. Yield 40\%

\section{Reactions of $\mathrm{XCo}(\mathrm{CO})_{2}\left(\mathrm{PPh}_{3}\right)_{2}$ with $\mathrm{Mg}\left(\mathrm{C}_{3} \mathrm{H}_{5}\right) \mathrm{Br}$}

A THF solution of $\mathrm{Mg}\left(\mathrm{C}_{3} \mathrm{H}_{5}\right) \mathrm{Br}$ prepared from $0.3 \mathrm{ml}$ of $\mathrm{C}_{3} \mathrm{H}_{5} \mathrm{Br}$ and an excess of $\mathrm{Mg}$ was added at $-15^{\circ} \mathrm{C}$ to $1.014 \mathrm{~g}(1.41 \mathrm{mmol})$ of $\mathrm{BrCo}(\mathrm{CO})_{2}\left(\mathrm{PPh}_{3}\right)_{2}$. The mixture was stirred at room temperature for $2 \mathrm{~h}$ to give an orange solution along with an insoluble residue. The solution was filtered, then evaporated to dryness, and the residue extracted into pentane. Evaporation of the solvent gave yellow crystals, which were characterized as $\left(\eta^{3}-\mathrm{C}_{3} \mathrm{H}_{5}\right) \mathrm{Co}(\mathrm{CO})_{2}\left(\mathrm{PPh}_{3}\right)$. Yield $20 \%$.

Reactions of $\mathrm{XCo}(\mathrm{CO})_{2}\left(\mathrm{PPh}_{3}\right)_{2}$ with $\mathrm{Na}\left(\mathrm{C}_{5} \mathrm{H}_{5}\right)$

Solid $\mathrm{ClCo}(\mathrm{CO})_{2}\left(\mathrm{PPh}_{3}\right)_{2}(1.839 \mathrm{~g}, 2.72 \mathrm{mmol})$ was added to a THF solution 
containing $2.72 \mathrm{mmol}$ of $\mathrm{Na}\left(\mathrm{C}_{5} \mathrm{H}_{5}\right)$. After $1.5 \mathrm{~h}$ stirring at room temperature the red solution was filtered, then evaporated under vacuum to leave an oil, the volatile material being collected in a liquid nitrogen trap. The presence of $\left(\eta^{5}-\mathrm{C}_{5} \mathrm{H}_{5}\right) \mathrm{Co}(\mathrm{CO})_{2}$ in this fraction was revealed by IR spectroscopy. The oil was extracted into pentane and the solution was chromatographed through alumina. Elution with pentane gave free $\mathrm{PPh}_{3}$, and a red solution was subsequently obtained by elution with toluene. $\left(\eta^{5}-\mathrm{C}_{5} \mathrm{H}_{5}\right) \mathrm{Co}(\mathrm{CO})\left(\mathrm{PPh}_{3}\right)$ was crystallized by evaporation from the solution. Yield $25 \%$.

Reactions of $\mathrm{XCO}(\mathrm{CO})_{2}\left(\mathrm{PPh}_{3}\right)_{2}$ with $\mathrm{P}(\mathrm{OMe})_{3}$

$\mathrm{P}(\mathrm{OMe})_{3}(0.60 \mathrm{ml}, 6.10 \mathrm{mmol})$ and $\mathrm{NaBPh}_{4}(0.437 \mathrm{~g}, 1.28 \mathrm{mmol})$ were added to a solution of $0.860 \mathrm{~g}(1.28 \mathrm{mmol})$ of $\mathrm{ClCo}(\mathrm{CO})_{2}\left(\mathrm{PPh}_{3}\right)_{2}$ in $\mathrm{CH}_{2} \mathrm{Cl}_{2}(60 \mathrm{ml})$. After $1 \mathrm{~h}$ stirring at room temperature the yellow solution was filtered, then evaporated to leave a yellow solid. This recrystallized from acetone to give crystalline $\{\mathrm{Co}(\mathrm{CO})$ $\left.\left[\mathrm{P}(\mathrm{OMe})_{3}\right]_{4}\right\}\left(\mathrm{BPh}_{4}\right)$. Yield $90 \%$.

The presence of $\mathrm{ClCo}(\mathrm{CO})_{2}\left[\mathrm{P}(\mathrm{OMe})_{3}\right]_{2}$ as an intermediate was confirmed when the reaction was monitored by IR spectroscopy.

Reactions of $\mathrm{XCo}(\mathrm{CO})_{2}\left(\mathrm{PPh}_{3}\right)_{2}$ with $\mathrm{AgClO}_{4}$ or $\mathrm{TlClO}_{4}$ and phosphines

$\mathrm{A} \mathrm{CH}_{2} \mathrm{Cl}_{2}$ solution of $0.50 \mathrm{~g}(0.74 \mathrm{mmol})$ of $\mathrm{ClCo}(\mathrm{CO})_{2}\left(\mathrm{PPh}_{3}\right)_{2}$ was treated with $0.15 \mathrm{~g}(0.74 \mathrm{mmol})$ of $\mathrm{AgClO}_{4}$ and the mixture was stirred at room temperature for 2 h. The insoluble solid was filtered off and hexane was added to the filtrate to give a pale-green precipitate. This was recrystallized from $\mathrm{CH}_{2} \mathrm{Cl}_{2}$ /ethanol to give pale yellow crystals of $\left[\mathrm{Co}(\mathrm{CO})_{3}\left(\mathrm{PPh}_{3}\right)_{2}\right] \mathrm{ClO}_{4}$.

The analogous reaction with $\mathrm{TlClO}_{4}$ took place more slowly, to give the same complex.

The same reaction with $\mathrm{TlClO}_{4}$ was carried out in the presence of $0.60 \mathrm{ml}(2.96$ mmol) of $\mathrm{PMePh}_{2}$ and the solution obtained was filtered. Addition of hexane gave an orange solid, which was washed with benzene and recrystallized from $\mathrm{CH}_{2} \mathrm{Cl}_{2}$ /hexane to give crystalline $\left[\mathrm{Co}(\mathrm{CO})_{2}\left(\mathrm{PMePh}_{2}\right)_{3}\right]\left(\mathrm{ClO}_{4}\right)$. Yield $85 \%$.

Analogous procedures were carried out using $1.44 \mathrm{mmol}$ of dppe or dppm. Orange crystals were obtained by recrystallization from $\mathrm{CH}_{2} \mathrm{Cl}_{2}$ /ethanol. They were characterized as $\left[\mathrm{Co}(\mathrm{CO})(\mathrm{L}-\mathrm{L})_{2}\right] \mathrm{CLO}_{4}$ with $\mathrm{L}-\mathrm{L}=$ dppe or dppm. Yield $80 \%$.

$X$-ray structure of $\mathrm{Co}(\mathrm{CO})(\mathrm{dppm})_{2} \mathrm{ClO}_{4}$

Crystal data: $\mathrm{C}_{51} \mathrm{H}_{44} \mathrm{O}_{5} \mathrm{ClCoP}_{4}, M=955.18$ monoclinic. Space group $P 2_{1} / c, a$ $17.895(6), b 10.751(2), c$ 24.687(4) $\AA, \beta$ 98.92(1) ${ }^{\circ} ; U$ 4692.0(1.9) $\AA^{3}, D_{\mathrm{x}} 1.352 \mathrm{~g}$ $\mathrm{cm}^{-3}$ for $Z=4, F(000)=1976$, Mo- $K_{\alpha}$ radiation $(\lambda 0.71069 \AA), \mu\left(\mathrm{Mo}-K_{\alpha}\right) 6.00$ $\mathrm{cm}^{-1}$.

\section{Intensity data collection and refinements}

A red single crystal of dimensions approximately $(0.12 \times 0.09 \times 0.12 \mathrm{~mm})$ was mounted on an Enraf-Nonius CAD-4 computer-controlled four circle diffractometer. Graphite-monochromated Mo- $K_{\alpha}$ radiation was used. Lattice parameters were determined by least-squares refinement of values for 25 reflections. Data collections were recorded by the $\omega / 2 \theta$ scan technique: $h,-22$ to $22 ; k, 0$ to $13 ; l, 0$ to 31 . Two standard reflections ( $15 \overline{5} 0,250)$ were checked after every 90 reflections, and no significant changes in intensities were detected. A total of 9680 reflections were 
collected in the range $0<2 \theta<30^{\circ}$, and 2656 for which $I \geqslant 3 \sigma(I)$ were used in further calculations. Intensities were corrected for Lorentz and polarization effects. No absorption corrections were made because of the small size of the crystal with a linear absorption coefficient $\mu\left(\mathrm{Mo}-K_{\alpha}\right)$ of $6.0 \mathrm{~cm}^{-1}$. No correction for extinction was made.

The structure was solved by a combination of direct methods, using the MULTAN program [26] and heavy atom methods to locate the cobalt, phosphorus and chlorine atoms. Other atoms were located by difference Fourier synthesis.

The structure was refined (on $F$ ) by full matrix least-squares calculations. All non-hydrogen atoms were defined anisotropically. In the later stages of the refinement $\mathrm{H}$ atoms were included at calculated positions, with fixed positions and with temperature factors equivalent to those of the atoms to which they were attached. During the refinement, considerable disorder in the $\mathrm{O}$ atoms in the perchlorate ion was revealed. On the basis of careful examination of difference syntheses around the $\mathrm{Cl}$ atom, which refined well, eight positions for the $\mathrm{O}$ atoms were found, and the anion was refined using a model involving these eight positions and refining the occupancy factors.

A final $R$ value of 0.077 and $R_{\mathrm{w}}=0.061$ (weighting scheme, empirical fit giving no trends in $\langle W \Delta 2 F\rangle$ vs. $\left\langle F_{0}\right\rangle$ and vs. $\left.\langle\sin \theta / \lambda\rangle\right)$ was obtained. The maximum residual electron density in the final difference Fourier synthesis was $0.57 \AA^{-3}$.

Anomalous dispersion corrections and atomic scattering factors were taken from International Tables for X-Ray Crystallography [27]. Calculations were performed with X-Ray system 80(28) and PARST(29) on a VAX-11 750 computer.

\section{Acknowledgements}

The authors greatly appreciate the financial support received from Comision Asesora de Investigación Científica y Técnica (Spain).Ref.2001-83.

\section{References}

1 C. Carriedo and P. Royo, An. Quim., in press.

2 W.J. Geary, Coord. Chem. Rev., 7 (1971) 81.

3 J.B. Orenberg, M.D. Morris and T, Veach long, Inorg. Chem:, 10 (1971) 933.

4 A. Sacco, Gazz. Chim. Ital., 93 (1963) 542.

5 M. Bressan, B. Corain, P. Rigo and A. Turco, Inorg. Chem., 9 (1970) 1733.

6 M. Aresta, M. Rossi and A. Sacco, Inorg. Chim. Acta, 3 (1969) 227

7 H. Hieber and H. Duchatsch, Chem. Ber., 98 (1965) 2530.

8 N. España, P. Gómez, P. Royo and A. Vázquez de Miguel, J. Organomet. Chem., 256 (1983) 141.

9 P.V. Rinze and U. Müller, Chem. Ber., 112 (1979) 1973.

10 T.S. Piper, F.A. Cotton and G. Wilkinson, J. Inorg. Nuc. Chem. 1 (1955) 165

11. R.B. King, Inorg. Chem., 5 (1966) 82.

12 L.W. Gosser, Inorg. Chem., 16 (1977) 430.

13 S. Attali and R. Poilblanc, Inorg. Chim. Acta, 6 (1972) 475

14 J. Peone and L. Vaska, Angew. Chem. Int. Ed., 10 (1971) 511.

15 A. Sacco and M. Freni, J. Inorg. Nucl. Chem., 8 (1958) 566.

16 P. Rigo, M. Bressan and A. Morvillo, J. Organomet. Chem., 105 (1976) 263.

17 C.F. Nobile, M. Rossi and A. Sacco, Inorg. Chim. Acta, 5 (1971) 698.

18 S. Barry, J. Chem. Phys., 32 (1969) 933

19 F.A. Cotton, K.I. Hardcastle and G.A. Rusholme, J. Coord. Chem., 2 (1973) 217

20 R.J. Puddephatt, Chem. Soc. Rev., 12 (1983) 99 
21 C. Bianchini, D. Masi, C. Mealli, A. Meli, M. Sabat and G. Scapacci, J. Organomet. Chem., 273 (1984) 91.

22 R.L. Harlow, R.J. McKinney and J.F. Witney, Organometallics, 2 (1983) 1839.

23 J.R. Bleeke and W.J. Peng, Organometallics, 3 (1984) 1422.

24 D. Boys, C. Escobar and S. Martinez-Carrera, Acta Cryst. B, 37 (1981) 351.

25 H. Endress, H.J. Keller, B. Müller and D. Schweitzer, Acta Cryst. C, 41 (1985) 607.

26 G. German, P. Main and M.M. Wollfson, Acta Cryst. A, 27 (1971) 368.

27 International tables for X-ray Crystallography, 1974, Coll. vol. IV. Birmingham, Kynoch Press.

28 S.M. Stewart (Ed.), The X RAY 80 system. Tech. Rep. TR-446, 1980, Computer Science Center, Univ. of Maryland, College Park, MD (U.S.A.).

29 M. Nardelli, Comput. Chem., 7 (1983) 95. 